\title{
Guest editor's introduction: cholangiopathies: review and update
}

\author{
Ann S. Fulcher \\ VCU Medical Center, Richmond, USA
}

The definitions of cholangiopathy range from broad to quite narrow. In its broadest and most generic sense, cholangiopathy is defined as any disease of the bile ducts. In contrast, cholangiopathy may be defined in more specific terms such as a category of chronic, progressive liver diseases that share a central target, the cholangiocyte, and lead to end-stage liver disease [1]. To further complicate matters, cholangiopathies include both neoplastic entities such as cholangiocarcinoma and nonneoplastic entities that range from the relatively common to the obscure. To simplify and focus this potentially wide-ranging discussion, I have chosen to concentrate on the non-neoplastic biliary entities that are most commonly encountered in clinical practice. To this end, this feature section includes review articles authored by wellknown experts addressing autoimmune diseases; overlap syndromes; iatrogenic and traumatic injuries of the bile ducts; recurrent pyogenic cholangitis; portal cholangiopathy; and congenital and acquired diseases of the pediatric biliary tract and gallbladder. Given that the imaging evaluation of biliary diseases has changed dramatically over the past two decades due to advances in $\mathrm{MR} / \mathrm{MRCP}, \mathrm{CT}$, and sonography, an update on appropriate imaging strategies is offered in each review article as appropriate.

In view of the increased awareness of autoimmunerelated biliary disease in general and the increasing prevalence of primary sclerosing cholangitis (PSC) in particular, Dr. Benjamin Yeh and colleagues address the imaging manifestations of the more commonly encountered of these entities including PSC, primary biliary cirrhosis, and IgG4-associated cholangitis (IAC). These authors emphasize the important role of imaging in corroborating the diagnosis in the case of PSC and IAC and in assessing for disease progression and the development of complications. In a related article, Dr. Venkatesh and colleagues review the so-called overlap

Correspondence to: Ann S. Fulcher; email: ann.fulcher@vcuhealth.org syndromes characterized by a subset of patients that demonstrate a combination of autoimmune biliary diseases.

The more frequent imaging evaluation of patients following trauma and surgical as well as non-surgical interventions has increased detection and heightened awareness of bile duct injuries. Dr. Soto and colleagues describe and illustrate the salient imaging features of a wide range of injuries resulting from penetrating and blunt trauma as well as from interventions such as laparoscopic cholecystectomy, hepatic resection, and hepatic transplantation. Treatment options for patients with bile duct injuries are presented.

Although the incidence of recurrent pyogenic cholangitis (RPC) has decreased in East Asia over the past several decades, RPC is being diagnosed with increasing frequency outside of East Asia due to an increase in immigrants from endemic areas. For this reason, radiologists worldwide must become familiar with the imaging features of RPC to avoid diagnostic errors. Dr. Cher Heng Tan shares his in-depth knowledge of RPC including its epidemiology, imaging features, and treatment options.

Despite portal cholangiopathy occurring in the vast majority of patients with portal vein thrombosis, this entity is not well known among radiologists and, as a result, is often underreported. Given that portal cholangiopathy may progress over time, become symptomatic and lead to severe complications such as secondary biliary cirrhosis, a review and update of this entity is indicated. In their review article, Drs. Winks and Moomjian describe predisposing factors, clinical manifestations, imaging features, imaging strategies, and potential mimics of portal cholangiopathy.

While some cholangiopathies occur both in children and adults, other cholangiopathies are identified primarily or exclusively in children. Likewise, while some congenital bile duct anomalies are initially identified in adulthood, many come to clinical attention during 
childhood. Therefore, Dr. Sharafinski and colleagues have contributed a review article that addresses both congenital and acquired diseases of the pediatric biliary tract and that offers suggestions for imaging aimed at minimizing radiation dose without compromising diagnostic accuracy.

It is my hope that these review articles will provide an update on some of the more commonly encountered, and in some cases under-recognized, cholangiopathies and will enhance radiologists' knowledge of these entities. I appreciate the kind invitation extended to me by Dr. Daniel Johnson, the Editor-in-Chief, to serve as guest editor for this feature section. My most sincere thanks goes to each of the contributing authors who generously shared their expertise and time.

\section{Reference}

1. Lazaridis KN, LaRusso NF (2015) The cholangiopathies. Mayo Clin Proc 90:791-800 